\title{
CHARACTER AMENABILITY AND CHARACTER PSEUDO-AMENABILITY OF CERTAIN BANACH ALGEBRAS
}

\author{
KOBRA OUSTAD
}

\begin{abstract}
In this paper, we study character amenability of semi-group algebras $\ell^{1}(S)$ and weighted semi-group algebras $\ell^{1}(S, \omega)$, for a certain semi-groups such as right(left) zero semigroup, rectangular band semi-group, band semi-group and uniformly locally finite inverse semi-group. In particular, we show that for a right (left) zero semi-group or a rectangular band semi-group, character amenability, amenability, pseudo - amenability of $\ell^{1}(S, \omega)$, for each weight $\omega$, are equivalent. We also show that for an Archimedean semi-group $S$, character pseudo - amenability, amenability, approximate amenability and pseudo-amenable of $\ell^{1}(S)$ are equivalent.
\end{abstract}

MSC(2010): Primary 58B34, 58J42, 81 T75.

Keywords: character amenability, character pseudo-amenable, rectangular band semigroup, Archimedean semi-group.

\section{Introduction}

The notions of amenability in Banach algebra was initiated by Jonson in [12]. Let $\mathfrak{A}$ be a Banach algebra and $E$ be a Banach $\mathfrak{A}$ - bimodule. We regards the dual space $E^{*}$ as a Banach $\mathfrak{A}$ - bimodule with the following module actions:

$(a . f)(x)=f(x . a),(f . a)(x)=f(a . x)\left(a \in \mathfrak{A}, f \in E^{*}, x \in E\right)$

For a Banach algebra $\mathfrak{A}$ the projective tensor product $\mathfrak{A} \widehat{\otimes} \mathfrak{A}$ is a Banach $\mathfrak{A}$-bimodule in a natural manner and the multiplication map $\pi: \mathfrak{A} \widehat{\otimes} \mathfrak{A} \longrightarrow \mathfrak{A}$ defined by $\pi(a \otimes b)=a b$ for $a, b \in \mathfrak{A}$ is a Banach $\mathfrak{A}$-bimodule homomorphism.

Amenability for Banach algebras introduced by B. E. Johnson [12]. Let $\mathfrak{A}$ be a Banach algebra and $E$ be a Banach $\mathfrak{A}$-bimodule. A continuous linear operator $D: \mathfrak{A} \longrightarrow E$ is a derivation if it satisfies $D(a b)=D(a) . b+a . D(b)$ for all $a, b \in \mathfrak{A}$. Given $x \in E$, the inner derivation $a d_{x}: \mathfrak{A} \longrightarrow E$ is defined by $a d_{x}(a)=a . x-x . a$. A Banach algebra $\mathfrak{A}$ is amenable if for every Banach $\mathfrak{A}$-bimodule $E$, every derivation from $\mathfrak{A}$ into $E^{*}$, the dual of $E$, is inner.

An approximate diagonal for a Banach algebra $\mathfrak{A}$ is a net $\left(m_{i}\right)_{i}$ in $\mathfrak{A} \widehat{\otimes} \mathfrak{A}$ such that $a . m_{i}-$ $m_{i} . a \longrightarrow 0$ and $a \pi\left(m_{i}\right) \longrightarrow a$, for each $a \in \mathfrak{A}$. The concept of pseudo-amenability introduced by F. Ghahramani and Y. Zhang in [9]. A Banach algebra $\mathfrak{A}$ is pseudo-amenable if it has an approximate diagonal. It is well-known that amenability of $\mathfrak{A}$ is equivalent to the existence of a bounded approximate diagonal. One may see $[15,16,18]$ for more details and related notions.

Date: Received: February 1,2021, Accepted: June 9, 2021. 
The notions of biprojectivity and biflatness of Banach algebras introduced by Helemski $\breve{i}$ in [10]. A Banach algebra $\mathfrak{A}$ is biprojective if there is a bounded $\mathfrak{A}$-bimodule homomorphism $\rho: \mathfrak{A} \longrightarrow \mathfrak{A} \widehat{\otimes} \mathfrak{A}$ such that $\pi o \rho=I_{\mathfrak{A}}$, where $I_{\mathfrak{A}}$ is the identity map on $\mathfrak{A}$. We say that $\mathfrak{A}$ is biflat if there is a bounded $\mathfrak{A}$-bimodule homomorphism $\rho: \mathfrak{A} \longrightarrow(\mathfrak{A} \widehat{\otimes} \mathfrak{A})^{* *}$ such that $\pi^{* *} o \rho=k_{\mathfrak{A}}$, where $k_{\mathfrak{A}}: \mathfrak{A} \longrightarrow \mathfrak{A}^{* *}$ is the natural embedding of $\mathfrak{A}$ into its second dual.

Kaniuth, Lau and Pym have introduced and studied in [14] and [13], the notion of $\varphi$ amenability for Banach algebras, where $\varphi: \mathfrak{A} \longrightarrow \mathbb{C}$ is a character. In [20], and also M. S. Monfared in [19] introduced the notion of character amenability for Banach algebras. Let $\mathfrak{A}$ be a Banach algebra over $\mathbb{C}$ and $\varphi: \mathfrak{A} \longrightarrow \mathbb{C}$ be a character on $\mathfrak{A}$, that is, an algebra homomorphism from $\mathfrak{A}$ in to $\mathbb{C}$, and let $\Phi_{\mathfrak{A}}$ denote the character space of $\mathfrak{A}$ (that is, the set of all character on $\mathfrak{A}$ ).In [19], see also [20], Monfared introduced the notion of character amenable Banach algebras. He called $\mathfrak{A}$ character amenable if it has a bounded approximate identity and it is $\varphi$ - amenable for all nonzero character $\varphi$ on $\mathfrak{A}$.

Character amenability for Banach algebras was introduced by Aghababa, Shi and Wu in [1].These notions have been studied for various classes of Banach algebras. For more details see, $[19,14,13,20]$ and [4]. As such character amenability is weaker than the classical amenability introduced by Johnson in [12], so all amenable Banach algebras are character amenable.

It is show in [15], that the character amenability of semi-group algebra $\ell^{1}(S)$ implies that the semi-group $S$ is amenable, and the authors focus on certain semi-groups such as inverse semigroup, Rees semi-group, Clifford semi-group and Brandt semi-group and study the character amenability of $\ell^{1}(S)$ in relation to the semi-group $S$.

Nasr-Isfahani and Nemati, in [22] introduced and studied a notion of character amenability based on the existence of a $\varphi$ - approximate digonal that is not necessarily bounded. They study character pseudo- amenability of certain Banach algebras.

Let $S$ be a semi-group and

$$
\ell^{1}(S)=\left\{f: S \rightarrow \mathbb{C},\|f\|_{1}=\sum_{s \in S}|f(s)|<\infty\right\} .
$$

We define the convolution of two elements $f, g \in \ell^{1}(S)$ by $(f * g)(s)=\sum_{u v=s} f(u) g(v)$, where $\sum_{u v=s} f(u) g(v)=0$, when there are no elements $u, v \in S$ with $u v=s$. Then $\left(\ell^{1}(S), *,\|\cdot\|_{1}\right)$ becomes a Banach algebra, that is called the semi-group algebra of $S$.

Let $S$ be a semi-group. A continuous function $\omega: S \longrightarrow(0, \infty)$ is a weight on $S$ if $\omega(s t) \leq \omega(s) \omega(t)$, for all $s, t \in S$. Then it is standard that

$$
\ell^{1}(S, \omega)=\left\{f=\sum_{s \in S} f(s) \delta_{s}:\|f\|_{\omega}=\sum_{s \in S}|f(s)| \omega(s)<\infty\right\}
$$

is a Banach algebra with the convolution product $\delta_{s} * \delta_{t}=\delta_{s t}$. These algebras are called Weighted convolution algebras.

In [17], the authors introduced the character pseudo- amenability of semi-group algebras. No much work has been done to date on the character amenability version for weighted semigroup algebra $\ell^{1}(S, \omega)$ on a semi-group $S$, as in the other notions for amenability.

It will be good to study this and see how the character amenability of $\ell^{1}(S, \omega)$ affects the structure of $S$. Thus, in this work, we study the character pseudo - amenability and character amenability of semi-group algebras and weighted convolution algebras on certain semi-groups. 


\section{Preliminaries}

We let $M_{\varphi_{r}}^{\mathfrak{A}}$ denote the class of Banach $\mathfrak{A}$ - bimodule $X$ for which the right module action of $\mathfrak{A}$ on $X$ is given by

$$
x . a=\varphi(a) x\left(a \in \mathfrak{A}, x \in X, \varphi \in \Phi_{\mathfrak{A}}\right),
$$

and $M_{\varphi_{l}}^{\mathfrak{A}}$ denote the class of Banach $\mathfrak{A}$-bimodule $X$ for which the left module action of $\mathfrak{A}$ on $X$ is given by

$$
a . x=\varphi(a) x\left(a \in \mathfrak{A}, x \in X, \varphi \in \Phi_{\mathfrak{A}}\right) .
$$

It is easy to see that the left module action of $\mathfrak{A}$ on the dual module $X^{*}$ is given by

$$
a . f=\varphi(a) f\left(a \in \mathfrak{A}, f \in X^{*}, \varphi \in \Phi_{\mathfrak{A}}\right) .
$$

Thus, we note that $X \in M_{\varphi_{r}}^{\mathfrak{A}}\left(\operatorname{resp} . X \in M_{\varphi_{l}}^{\mathfrak{A}}\right)$ if and only if $X^{*} \in M_{\varphi_{l}}^{\mathfrak{A}}\left(\operatorname{resp} \cdot X^{*} \in M_{\varphi_{r}}^{\mathfrak{A}}\right)$.

Let $\mathfrak{A}$ be a Banach algebra and let $\varphi \in \Phi_{\mathfrak{A}}$, we recall from [20] and [19] that

i) $\mathfrak{A}$ is left $\varphi$-amenable if every continuous derivation $D: \mathfrak{A} \longrightarrow X^{*}$ is inner for every $X \in M_{\varphi_{r}}^{\mathfrak{A}}$

ii) $\mathfrak{A}$ is right $\varphi$-amenable if every continuous derivation $D: \mathfrak{A} \longrightarrow X^{*}$ is inner for every $X \in M_{\varphi_{l}}^{\mathfrak{A}}$;

iii) $\mathfrak{A}$ is left character amenable if it is left $\varphi$-amenable for every $\varphi \in \Phi_{\mathfrak{A}}$;

iv) $\mathfrak{A}$ is right character amenable if it is right $\varphi$-amenable for every $\varphi \in \Phi_{\mathfrak{A}}$;

$v) \mathfrak{A}$ is character amenable if it is both left and right character amenable.

We also recall the following defintions from that, for $\varphi \in \Phi_{\mathfrak{A}}$, a left (right) $\varphi$-approximate digonal for $\mathfrak{A}$ is a net $\left(m_{\alpha}\right)$ in $\mathfrak{A} \otimes \mathfrak{A}$ such that

$$
\begin{gathered}
(i)\left\|m_{\alpha} \cdot a-\varphi(a) m_{\alpha}\right\| \rightarrow 0\left(\left\|a . m_{\alpha}-\varphi(a) m_{\alpha}\right\| \rightarrow 0\right)(a \in \mathfrak{A}) ; \\
(i i)\left\langle\varphi \otimes \varphi, m_{\alpha}\right\rangle=\varphi\left(\pi\left(m_{\alpha}\right)\right) \rightarrow 1,
\end{gathered}
$$

where $\pi: \mathfrak{A} \otimes \mathfrak{A} \rightarrow \mathfrak{A}$ defined by $\pi(a \otimes b)=a b(a, b \in \mathfrak{A})$ is the product map. The notion of $\varphi$-approximate digonal was introduced and studied by Hu, Monfared and Traynor [20]. Let $\mathfrak{A}$ be a Banach algebra and $\varphi \in \Phi_{\mathfrak{A}}$. We recall from [21] that

(i) $\mathfrak{A}$ is $\varphi$-pseudo-amenable if there is a $\varphi$-approximate digonal for $\mathfrak{A}$;

(ii) $\mathfrak{A}$ is character pseudo - amenable if $\mathfrak{A}$ has a right approximate identity and it is $\varphi$-pseudoamenable for all $\varphi \in \Phi_{\mathfrak{A}}$.

\section{Character amenability of semi-group algebras}

In the section, we prove some general results for semi-group algebras. It is clear that every biprojective Banach algebra is biflat. Also we recall that a Banach algebra $\mathfrak{A}$ is amenable if and only if it is biflat and has a bounded approximate identity. By [19, Theorem 2.6], If $\mathfrak{A}$ is character amenable, then $\mathfrak{A}$ has a bounded approximate identity. So we can have the following results.

A semi-group $S$ is a left zero semi-group if $s t=s$, and it is a right zero semi-group if $s t=t$ for each $s, t \in S$.

Proposition 3.1. Suppose that $S$ is a right (left) zero semi-group. Then $\ell^{1}(S)$ is character amenable if and only if it is amenable. 
proof: From [6, proposition 3.1], $\ell^{1}(S)$ is biflat. Since $\ell^{1}(S)$ is character amenable, thus $\ell^{1}(S)$ has a bounded approximate identity. By the above argument, $\ell^{1}(S)$ is amenable.

Let $S$ be a semi-group and let $E(S)=\left\{p \in S: p^{2}=p\right\}$. We say that $S$ is a band semi-group if $S=E(S)$. A band semi-group $S$ satisfying $s t s=s$, for each $s, t \in S$ is called a rectangular band semi-group.

Corollary 3.2. Let $S$ be a rectangular band semi-group. Then $\ell^{1}(S)$ is character amenable if and only if it is amenable.

proof For a rectangular band semi-group $S$, it is known that $S \simeq L \times R$, where $L$ and $R$ are left and right zero semi-groups, respectively [11, Theorem 1.1.3]. So,

$$
\ell^{1}(S) \cong \ell^{1}(L \times R) \cong \ell^{1}(L) \otimes \ell^{1}(R) .
$$

Since $\ell^{1}(S)$ is character amenable, now by [1, proposition 6.3], $\ell^{1}(L)$ and $\ell^{1}(R)$ are character amenable. From proposition 3.1, it follows that $\ell^{1}(L)$ and $\ell^{1}(R)$ are amenable, so $\ell^{1}(S)$ is amenable.

Corollary 3.3. Let $S$ be a rectangular band semi-group. Then the following are equivalent:

(i) $\ell^{1}(S)$ is character amenable.

(ii) $\ell^{1}(S)$ is amenable.

(iii) $S$ is singleton.

proof From corollary 3.2, $(i)$ and $(i i)$ is equivalent and also by [6, theorem 3.3], (ii) and (iii) are equivalent.

Let $S$ be a band semi-group. Then by [11, Theorem 4.4.1], $S$ is a semilattice of rectangular band semi-groups. Indeed, $S=\cup_{\alpha \in Y} S_{\alpha}$ where $Y=\frac{S}{\tau}$ and for each $\alpha=[s] \in Y, S_{\alpha}=[s]$.

Theorem 3.4. Suppose that $S$ be a band semi-group. If $\ell^{1}(S)$ is character amenable, then $S$ is a finite semilattice.

proof: By the above argument, let $S=\cup_{\alpha \in Y} S_{\alpha}$ is a semilattice of rectangular band semigroups and $\ell^{1}(S)$ is character amenable. Indeed, we have $S_{\alpha} \cdot S_{\beta} \subseteq S_{\alpha \beta}$ for each $\alpha, \beta \in Y$. It follows that $\ell^{1}(S)$ is $\ell^{1}$-graded of $\left(\ell^{1}\left(S_{\alpha}\right)\right)$ 's over the semilattice $Y$. Indeed, we have

$$
\ell^{1}(S) \cong \bigoplus_{\alpha \in Y} \ell^{1}\left(S_{\alpha}\right)
$$

By [1, proposition 6.3], $\ell^{1}\left(S_{\alpha}\right)$ is character amenable and $Y$ is finite. Since each $S_{\alpha}$ is rectangular band semi-group, so by corollary $3.3, S_{\alpha}$ is singleton for each $\alpha \in Y$. So $S$ is isomorphic to $Y$. Thus, $S$ is a semilattice .

Corollary 3.5. Let $S$ be a uniformly locally finite band semi-group. Then, the following are equivalent:

(i) $\ell^{1}(S)$ is character amenable.

(ii) $S$ is a finite semilattice.

(iii) $\ell^{1}(S)$ is approximately amenable.

(iv) $\ell^{1}(S)$ is amenable.

proof: $(i) \rightarrow(i i)$, by theorem 3.4 and $(i i) \longleftrightarrow(i i i) \longleftrightarrow(i v)$ from [26, corollary 4.9] and also $(i v) \longleftrightarrow(i)$, is clear. 
3.1. Character amenability of weighted semi-group algebras. In this section, we extend the results for $\ell^{1}(S)$ to the weighted case $\ell^{1}(S, \omega)$. For $f, g \in \ell^{1}(S, \omega)$, it is obvious that $f * g=\varphi_{S}(f) g$ if $S$ is a right zero semi-group, and $f * g=\varphi_{S}(g) f$ if $S$ is a left zero semi-group, where $\varphi_{S}$ is the augmentation character on $\ell^{1}(S, \omega)$.

Proposition 3.6. Suppose that $S$ is a right (left) zero semi-group and $\omega$ be a weight on $S$. Then $\ell^{1}(S, \omega)$ is character amenable if and only if $S$ is singleton.

proof : From [23, Proposition 2.1], $\ell^{1}(S, \omega)$ is biflat. Since $\ell^{1}(S, \omega)$ is character amenable so it has a bounded approximate identity and thus is amenable. Now it is immediate by [23, Proposition 2.5].

Corollary 3.7. Let $S$ be a right (left) zero semi-group and $\omega$ be a weight on $S$. Then the following are equivalent:

(i) $\ell^{1}(S, \omega)$ is character amenable.

(ii) $S$ is singleton.

(iii) $\ell^{1}(S, \omega)$ is pseudo-amenable.

(iv) $\ell^{1}(S, \omega)$ is amenable.

Proof. By proposition 3.6 and [23, corollary 2.8] is clear.

Theorem 3.8. Let $S$ be a rectangular band semi-group and $\omega$ be a weight on $S$. Then $\ell^{1}(S, \omega)$ is character amenable if and only if $S$ singleton.

Proof: suppose that $\ell^{1}(S, \omega)$ be character amenable, by [18, Theorem 3.4], has a bounded approximate identity and by [23, proposition 2.3] is biflat. So, [23, Theorem 2.4] completes the proof.

The following is a combination of Theorems 3.8 and [23, corollary 2.9].

Corollary 3.9. Let $S$ be a rectangular band semi-group, and let $\omega$ be a separable weight on $S$. Then the following are equivalent:

(i) $\ell^{1}(S, \omega)$ is character amenable.

(ii) $S$ is singleton.

(iii) $\ell^{1}(S, \omega)$ is pseudo-amenable.

(iv) $\ell^{1}(S, \omega)$ is amenable.

Let $(P, \leq)$ is a partially ordered set. Then $(P, \leq)$ is locally finite if $(x]=\{y \in S: y \leq x\}$ is finite for every $x \in S$, and it is uniformly locally finite if $\sup \{|(x]|: s \in S\}<\infty$.

We recall that a semi-group $S$ is an inverse semi-group if for each $s \in S$ there exists a unique element $s^{*} \in S$ with $s s^{*} s=s$ and $s^{*} s s^{*}=s^{*}$.

Let $S$ be an inverse semi-group. We define an equivalence relation $D$ on $S$ by $s D t$ if and only if there exists $x \in S$ such that $s^{*} s=x x^{*}$ and $t^{*} t=x^{*} x$. Let $\left\{D_{\lambda}: \lambda \in \Lambda\right\}$ be the collection of all $D_{-}$classes of $S$. For each $p_{\lambda} \in E\left(D_{\lambda}\right)$, the maximal subgroup of $S$ at $p_{\lambda}$ is denoted by $G_{p_{\lambda}}$. It is easily verified that $G_{p_{\lambda}}=\left\{s \in S: s s^{*}=s^{*} s=p_{\lambda}\right\}$.

The following result is Theorem 2.3 and Corollary 2.5 of [7].

Theorem 3.10. Let $S$ be a semi-group and $\omega$ be a weight on $S$.

(i) If $\omega \geq 1$ and $\ell^{1}(S, \omega)$ is character amenable, then $\ell^{1}(S)$ is character amenable.

(ii) If $\omega \leq 1$ and $\ell^{1}(S)$ is character amenable, then $\ell^{1}(S, \omega)$ is character amenable 
Corollary 3.11. Let $S=M^{0}(G, I)$ be the Brandt semi-group and $\omega$ be a weight on $S$. Then the following are equivalent:

(i) $\ell^{1}(S, \omega)$ is character amenable.

(ii) $\ell^{1}(S)$ is character amenable.

(iii) $I$ is finite and in the case where $|I|=1$ then $G$ is amenable.

We now consider character amenability of $\ell^{1}(S, \omega)$, where $S$ is a uniformly locally finite inverse semi-group and $\omega \geq 1$.

Theorem 3.12. Let $S$ be a uniformly locally finite inverse semi-group and $\omega \geq 1$ be a weight on $S$. Then $\ell^{1}(S, \omega)$ is character amenable, if and only if $E(S)$ is finite and $G_{p_{\lambda}}$ is amenable for each $\lambda \in \Lambda$ with $\left|E\left(D_{\lambda}\right)\right|=1$.

proof: Let $\ell^{1}(S, \omega)$ be character amenable, By theorem 3.10, $\ell^{1}(S)$ is character amenable, so by [5, theorem 2.6] is clear. Conversely, since $E(S)$ is finite and $S$ is inverse, then $S$ has a principal series

$$
S=S_{1} \supset S_{2} \supset S_{3} \supset \ldots \supset S_{m-1} \supset S_{m}=K(S)
$$

of ideals of $S$, where $K(S)$ is the minimum ideal, see [19, theorem 3.12]. $\frac{S_{i}}{S_{i+1}}$ is a simple inverse semi-group with a finite number of idempotents, and so is a group. Also, for $i=1,2, \ldots, n-1$ , $\frac{S_{i}}{S_{i+1}}$ is 0 -simple with a finite number of idempotents, and so is a completely 0-simple inverse semi-group, that is a Brandt semi-group. By corollary 3.11, $\ell^{1}(S, \omega)$ is character amenable if and only if $\ell^{1}(S)$ is character amenable and by proof of [15, proposition 3.1], $\ell^{1}(S)$ is character amenable if and only if $\ell^{1}\left(\frac{S_{i}}{S_{i+1}}\right)$ is character amenable for $i=1,2, \ldots, n-1$. For $i=1,2, \ldots, n-1$, let $G_{i}$ be the group of the Brandt semigrop $\frac{S_{i}}{S_{i+1}}$ and $\ell^{1}\left(\frac{S_{i}}{S_{i+1}}\right)$ is amenable if $G_{i}$ is amenable for $i=1,2, \ldots, n-1$. So $\ell^{1}(S, \omega)$ is character amenable if $G_{i}$ is amenable and the groups $G_{i}$ are maximal subgroups of $S$.

Corollary 3.13. Let $S$ be a uniformly locally finite semilattice and $\omega \geq 1$ be a weight on $S$. Then $\ell^{1}(S, \omega)$ is character amenable, if and only if $S$ is finite.

proof: Suppose that $\ell^{1}(S, \omega)$ is character amenable, then by theorem 3.10 and [5, theorem 2.6] is clear.

Conversely, since $S$ is finite, $\ell^{1}(S) \cong \ell^{1}(S, \omega)$ and $\ell^{1}(S)$ is finite-dimensional. Then by [5, corollary 2.8], $\ell^{1}(S)$ is character amenable so $\ell^{1}(S, \omega)$ is character amenable.

\section{Character pseudo - amenability of semi-group algebras}

In this section, we would like to present a class of commutative semi-groups $S$ which character pseudo-amenability and approximate amenability over $\ell^{1}(S)$ are equivalent. Recall that a semi-group $S$ is Archimedean if $S$ is commutative and for each $s, t \in S$ there exists $n \in \mathbb{N}$ such that

$$
s^{n} \in t S=\{t u: u \in S\} .
$$

Theorem 4.1. Let $S$ be an Archimedean semi-group. If $\ell^{1}(S)$ is character pseudo-amenable, then for each $s, t \in S, S s=S t$ and $S$ has an idempotent element.

Proof. Suppose that $\ell^{1}(S)$ is character pseudo-amenable. By definition of character pseudo - amenability, $\ell^{1}(S)$ has a right approximate identity and so $\overline{\ell^{1}(S)^{2}}=\ell^{1}(S)$. We know that $\ell^{1}(S t)$ is a complemented and so $\ell^{1}(S t)$ is weakly complemented ideal of $\ell^{1}(S)$.It follows that 
$\ell^{1}(S t)$ has a right approximate identity. Now, we conclude that $\overline{\ell^{1}(S t)^{2}}=\ell^{1}(S t)$ and so $S t S t=S t$. This implies that $S t^{m}=S t$ for each $t \in S$ and $m \in \mathbb{N}$. Fix two element $s, t \in S$. Since $S$ is Archimedean, there is $n \in \mathbb{N}$ such that $s^{n} \in S t$. Thus, we have $S s \subseteq S t$ and so we have $S s=S t$.

To prove the second part, we use the result of the previous part. So we conclude $S=S^{2}=$ $\cup_{t \in S} S t$ and $S=S t$ for all $t \in S$. Fix an element $t \in S$. There exist $u, v \in S$ such that $t=u t$ and $u=v t$. Thus,

$$
u^{2}=v t v t=v u t=v t=u
$$

and the proof is complete.

In the sequel, we show that if $S$ is Archimedean semi-group, then character pseudoamenability, pseudo- amenability, amenability and approximate amenability for $\ell^{1}(S)$ are equivalent.

Theorem 4.2. Let $S$ be an Archimedean semi-group. Then the following are equivalent:

(i) $\ell^{1}(S)$ is character pseudo-amenable.

(ii) $S$ is an amenable group.

(iii) $\ell^{1}(S)$ is pseudo-amenable.

(iv) $\ell^{1}(S)$ is amenable.

$(v) \ell^{1}(S)$ is approximately amenable.

Proof. $(i) \rightarrow($ ii $)$ Let $\ell^{1}(S)$ is character pseudo-amenable. By theorem 4.1, for $u \in E(S)$, we have $S=S u$. It follows that $u$ is the identity element of $S$. Now, if $t s=k s$ then exists $v \in S$ such that $u=s v$. Thus,

$$
t=t u=t s v=k s v=k u=k .
$$

Therefore $S$ is cancellative and by [17, corollary 4.6], $S$ is amenable group.

$($ ii $) \longleftrightarrow(i i i) \longleftrightarrow(i v) \longleftrightarrow(v)$ follows from [27, theorem 2.3].

$(v) \rightarrow(i)$ follows from [22, corollary 2.6].

\section{REFERENCES}

[1] H. Pourmahmood- Aghababa, L.Y. Shi and Y. J. Wu, Generalized notions of character amenability, Acta Mathematica Sinica, English series, 29, Issue 7, (2013), 1329-1350.

[2] H. G. Dales, Banach algebras and automatic continuity, London Mathematical Society Monographs, New Series, Volume 24, The Clarendon Press Oxford, 2000.

[3] H. G. Dales,A. T. Lau and D. Strauss, Banach algebras on semi-groups and their compactifications, Mem. Amer. Math.Soc,205, 2010.

[4] G. H. Esslamzadeh. Banach algebra structute and amenability of a class of matrix algebra with application. J. Functional Analysis.161 (1999). 361-383.

[5] M. Essmaili, M. Filali, $\phi$ - Amenability and character amenability of some classes of Banach algebras, Houston Journal of Mathematics, Vol. 39, No. 2(2013). 515-530.

[6] M. Essmaili and A. Medghalchi, Biflatness of certain semi-group algebra, Bull. Iran. Math. Soc., 39 (2013), 959-969.

[7] M. Essmaili, M. Rostami, Amenability of weighted semi-group algebrs based on character. The 46th Annual Iranian Mathematics Conference,2015.

[8] M . Essmaili, M. Rostami, and A . R . Medghalchi, Pseudo-contractibility and Pseudo-amenability of semi-group algebrs, Arch . Math.97(2011), 167-177.

[9] F. Ghahramani and Y. Zhang, Pseudo-amenable and Pseudo-contractible Banach algebras, Math. Proc. Cambridge Phil. Soc., 142 (2007), 111-123.

[10] A. Ya. Helemski $\breve{i}$, Flat Banach modules and amenable algebras, Trans. Moscow Math. Soc. 47 (1985), 199-224. 
[11] J. M. Howie, Fundamentals of semi-group theory, London Mathematical Society Monographs, Volume 12, The Clarendon Press Oxford, 1995.

[12] B.E. Johnson, Cohomology in Banach algebras, Mem . Amer. Math. Soc.127 (1972).

[13] E. Kaniuth, A. T. Lau and J. S. Pym, On character amenability of Banach algebras, J, Math. Anal. Appl. 344 (2008), 942-955.

[14] E. Kaniuth, A. T. Lau and J. S. Pym, On $\varphi$-amenaibility of Banach algebras, Math. Proc. Cambridge philos. Soc. 144 (2008), 85-96.

[15] S. M. Maepa and O. T. Mewomo, On character Amenability of semi-group algebrs, Quaestiones Mathematices, (2015) , 307-318.

[16] O. T. Mewomo and S. M. Maepa, On character Amenability of Beurling and second dual algebras, Acta Universitatis Apulensis, No. 38/2014, 67-80.

[17] O. T. Mewomo, A. A. Mebawondu, U. O. Adiele, P. O. Olanipekun, On character pseudo-amenability semi-group algebras, Proceedings of the Jangjeon Mathematical Society, 20 (2017), No .4. pp. 583-593.

[18] O. T. Mewomo, Note On character amenability in Banach algebras, Math. Reports ,19 (69), 3 (2017), 293-312.

[19] M. S. Monfared, Character amenability of Banach algebras, Math. Proc. Cambridge philos. Soc. 144 (2008), 697-706.

[20] Z. Hu, M. S. Monfared and T. Traynor, On character amenable of Banach algebra, Studia Math. 193 (1) (2009), 53-78.

[21] R. Nasr-Isfahani and M. Nemati, Character pseudo-amenability of Banach algebras, Colloquium Mathematicum. 132 (2013), 177-193.

[22] R. Nasr-Isfahani and M. Nemati, Cohomological Characterization of Character pseudo-amenability Banach algebras, Bull. Aust. Math. Soc. 84 (2011), 229-237.

[23] K. Oustad, A, Mahmoodi, Pseudo-amenability of weighted semi-group algebrs, Journal of mathematical Extension, Vol. 14, No. 4, (2020),91-99.

[24] A. L. T. Paterson, Amenability, Mathematical Surveys and Monographs,Number 29, American Mathematical Society, Providence, RI, 1988.

[25] P. Ramsden, Biflatness of semi-group algebras, semi-group Forum.79 (2009) , 515-530.

[26] M. Rostami , A. Pourabbas , M. Essmaili , Approximate amenability of certain inverse semi-group algebras, Acta Mathematica Scientia 2013, 33 B (2) ,565-577.

[27] M. Soroushmehr .M. Rostami. M. Essmaili, On pseudo-amenability of commutative semi-group algebras and their second duals, semi-group Forum, Springer Science+Business Media, LLC (2017).

[28] J. Zhang, Character amenable Banach algebras, University of windsor, Scholarship at UWindsor, 2012.

(Kobra Oustad) Dehdasht of mathematics, Dehdasht Branch, Islamic Azad University, DeHDASHT, IRAN.

Email address: ostad.kobra@gmail.com 